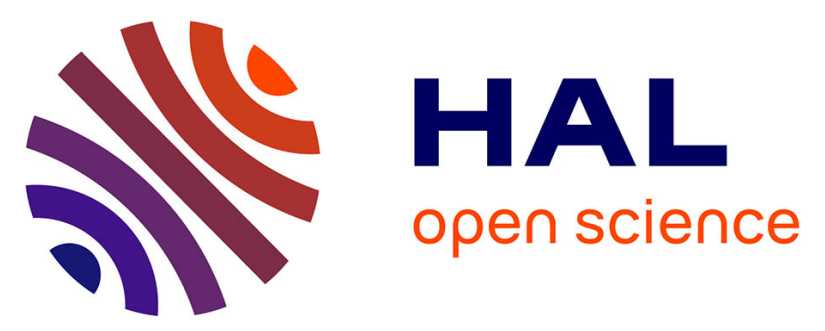

\title{
Design and Evaluation of a Novel Haptic Interface for Horse-Drawn Carriage Simulation
}

Jean-Rémy Chardonnet, Julien Ryard, Melanie Secheppet, Clémence Bénézet, Guillaume Azéma, Serge Leblanc

\section{- To cite this version:}

Jean-Rémy Chardonnet, Julien Ryard, Melanie Secheppet, Clémence Bénézet, Guillaume Azéma, et al.. Design and Evaluation of a Novel Haptic Interface for Horse-Drawn Carriage Simulation. IEEE Transactions on Haptics (ToH), 2021, 14 (3), pp.491-502. 10.1109/toh.2021.3052151 . hal-03366249

\author{
HAL Id: hal-03366249 \\ https://hal.science/hal-03366249
}

Submitted on 5 Oct 2021

HAL is a multi-disciplinary open access archive for the deposit and dissemination of scientific research documents, whether they are published or not. The documents may come from teaching and research institutions in France or abroad, or from public or private research centers.
L'archive ouverte pluridisciplinaire HAL, est destinée au dépôt et à la diffusion de documents scientifiques de niveau recherche, publiés ou non, émanant des établissements d'enseignement et de recherche français ou étrangers, des laboratoires publics ou privés. 


\title{
Design and Evaluation of a Novel Haptic Interface for Horse-Drawn Carriage Simulation
}

\author{
Jean-Rémy Chardonnet, Member, IEEE, Julien Ryard, Mélanie Secheppet, Clémence Bénézet, Guillaume Azéma, \\ and Serge Leblanc
}

\begin{abstract}
Animal welfare has become an increasingly important concern in the sports field. Learning horse-drawn carriage driving requires much time and effort for both the drivers and the horses because the associated gestures to avoid harming the horses are difficult to acquire. This raises the need to develop realistic simulation environments for future drivers. To this end, two haptic interface prototypes were designed, coupled with dedicated simulation software. The first was developed based on a SPIDAR haptic device and implemented simple behaviors of the carriage. A user study demonstrated interest in such a simulator, which led to the design of a second prototype, on a different architecture than the first prototype, for integrating more precise laws of horse behavior such as mood and allowing a more subtle control of forces. An evaluation with driving learners revealed that the simulator was capable of not only producing sensations close to reality but also improving the interaction between the trainer and the learner.
\end{abstract}

Index Terms-Force feedback, horse-drawn carriage simulation, training, design.

\section{INTRODUCTION}

\section{A. Background}

D RIVING a horse-drawn carriage can be a high-level activity in which horses are attached to a carriage. Horses are driven using reins held by drivers seated on the carriage. There has been growing interest in horse-drawn carriage driving for several years: (a) sports competitions bring increasing popularity and arouse new desires for practice, (b) equestrian sports and leisure professionals are willing to diversify their range of activities, and (c) horse-drawn vehicles (agriculture, forestry, viticulture, horticulture, and service activities in towns, such as tourism, school transportation, maintenance of green areas, waste sorting, and so on) have become (again) trendy, reconciling sustainability and the defense of cultural heritage. This activity thus requires qualified staff.

In this frame, it is important to allow the majority to practice this activity in the best conditions; today, it appears that these conditions are perfectible. In a dynamic environment, a horse-drawn carriage is indeed an on-board piloting activity involving the cooperation of one or several horses and aiming

J.-R. Chardonnet and J. Ryard are with Arts et Métiers Institute of Technology, LISPEN, HESAM Université, 71100 Chalon-sur-Saône, France. E-mail: \{jean-remy.chardonnet,julien.ryard\}@ensam.eu.

M. Secheppet, G. Azéma and S. Leblanc are with LIRDEF EA3749, UM-UPVM, 34092 Montpellier, France. E-mail: \{melanie.secheppet,guillaume.azema,serge.leblanc\}@umontpellier.fr

C. Bénézet is with Ifce - Haras national d'Uzès, 30700 Uzès, France. Email: clemence.benezet@ifce.fr

This work was supported by the Ifce and SAHn under grant no.

2016-17-007. to optimize a trajectory with respect to the drivers, the environment and the potential surrounding users. In France especially, the expertise in horse-drawn carriage driving is rather found in renowned drivers whose exceptional knowledge (built for many years of involvement and patient attention) is little captured, lowly diffused in training courses intended primarily for a public of enlightened amateurs, and jeopardized by time. This in particular is a critical issue as driving (for technical and equipment reasons and safety reasons) is a complex and difficult activity to teach, especially regarding the communication between the horses and the driver through the reins [1]. Indeed, during training, drivers must learn to manipulate the reins they hold to achieve the desired trajectories with a precise behavior from the horses. Today, this training can be very tedious, as it is mandatory to equip a carriage and several horses. Moreover, it is impossible to solicit horses indefinitely for training. Furthermore, improper treatment can seriously injure horses, especially with novice drivers.

To limit the tiredness, stress and risks for the driver and the horses, simulation tools are used. In the specific literature on the teaching of horse-drawn carriages, weight simulators must be a regularly employed tool in driver training to educate their hands [2], [3]. Teachers develop creativity in designing and using these traditional simulators; however, these tools are not interactive. A more appropriate alternative is the use of computer-based simulators that employ visual and haptic tools. Computer-based simulators provide the benefits of being interactive, more flexible, and having the possibility to design and repeat scenarios ad infinitum. In addition, it is possible to adapt simulations to the level of the trainees, allowing for smooth progression during training. However, existing simulators do not comply with the criteria imposed by the field in terms of interaction accuracy and simulation realism.

\section{B. Horse-Drawn Carriage Simulators}

Several equestrian simulators can be found in the literature. Apart from simulators dedicated to leisure (amusement parks, among others), we can cite the PERSIVAL [4] and the Clergerie [5] simulators or the simulator proposed in [6]. Simulators based on parallel robots were designed for equestrian gait simulation, as well [7]. However, these simulators are dedicated to riders, aiming at making them conscious of a horse's gait by replicating these movements as realistically as possible. Furthermore, a large part of equestrian simulators was developed for healthcare applications [8], [9]. However, in the frame of horse-drawn carriage simulation, this type 
of simulator does not provide significant $i$ nterest, $\mathrm{s}$ ince the movements in horseback riding are not identical to those in horse-drawn carriage driving.

To our knowledge, horse-drawn carriage simulators are relatively rare. The simplest versions are static and made of inert reins passing through rings affixed to a wall in front of the rider. These reins are then tightly maintained through a suspended mass. Though rudimentary, these so-called weight simulators are used in the first s teps of learning horse-drawn carriage gestures [3]. However, this type of simulator does not provide any feedback on potential horse behaviors and cannot reliably reflect the forces i nvolved in a real situation, as the mass never varies by time. Vennetier proposed a horsedrawn driving simulator equipped with a haptic system that uses motors acting on reins, which aim to simulate the forces that occur during driving [10]. Visual feedback of the path to be followed and of the user's actions is provided through a visual display. For each rein, lights embedded in the display indicate directional changes. Encke implemented a haptic device that allowed for providing close-to-real sensations by reproducing an articulated horse head on which the reins were directly fixed [11]. This d evice a llows the d river to be conscious of horse movements during the simulation; however, these devices propose to apply only relatively constant forces, despite integrating force feedback, and thus do not allow for developing ad libitum scenarios, especially to simulate critical situations, and do not ensure the repeatability of the movements.

To include realistic virtual environments in the simulation, past work developed the animation of virtual horse skeletons performing different gaits (walk, trot and canter) [12], [13]. In our context, as the driver is sitting behind the horse, the driver's viewpoint makes it unnecessary to produce animations of the movements of the virtual horse. Game editors released numerous video games pertaining to horse-drawn carriage driving, such as PK Horse Carriage Simulator ${ }^{1}$ and Horse Cart Carriage Farming Transport Simulator ${ }^{2}$. However, these games are purely recreational and therefore do not integrate any of the issues that we address here. Additionally, no haptic feedback is provided through these games.

The primary drawbacks of current horse-drawn carriage simulators for realistic simulation are as follows:

- highly basic mechanical systems,

- lack of variable force feedback (depending on the situations or on the horse movements),

- lack of relevant visual feedback for learning drivers.

To satisfy the need for interaction and simulation fidelity, we designed a novel horse-drawn carriage simulator integrating haptic feedback that we interfaced using in-house real-time simulation software. We developed two prototypes that we evaluated using both novice and experienced drivers. This paper presents the design, the development of the haptic feedback and the evaluation of these prototypes. This simulator

\footnotetext{
${ }^{1}$ https://www.microsoft.com/en-us/p/pk-horse-carriage-simulator/ 9nblggh $4 \times 8 \mathrm{q} 8$

${ }^{2}$ https://play.google.com/store/apps/details?id=com.tgs.village.horsecart. transport.simulator $3 \mathrm{~d} \& \mathrm{hl}=\mathrm{en} \_\mathrm{US}$
}

does not aim to completely replace training via real horses or theoretical courses. Rather, it serves as a complementary tool to other teaching tools such as videos. Its goal is to allow to learn movements faster than one would with real horses.

\section{General CONCEPT}

The specificity of horse-drawn carriage driving is to involve a highly non-deterministic system that is the horse. If horses are trained to cooperate with drivers and thus their behaviors are more or less known, reproducing such behaviors in simulation remains a challenge.

The driver's movements result from the energy provided by one or more horses. The safety and quality of the trajectories produced are largely linked to the availability of the horses and the quality of their locomotion. The driver therefore constantly seeks cooperation and close and precise communication with his or her horses. While handling the carriage, the driver can perform several movements that can have highly variable amplitudes, from several centimeters to nearly one meter, at various frequencies. Furthermore, the difficulty in horse-drawn carriage driving is the ability to communicate with the horse(s) mainly through the reins and especially maintain what is called "contact" between the horse and the driver. Contact is defined as the link between the driver's aids (here the reins) and the horse's body, primarily between the horse's mouth and the driver's hand. According to professional drivers, contact typically involves two main factors, namely the weight (which may range "between a PET bottle cap and a six-pack of bottled water", or between $\pm 10 \mathrm{~g}$ and $\pm 10 \mathrm{~kg}$ ) and the tension in the reins (which can be "hard or soft"). These parameters must be considered when selecting the components of the simulator. Moreover, as this notion of contact is highly difficult to describe efficiently to trainees, the simulator must allow to learn by doing being as close to reality as possible, based on a well-known principle called procedural memory [14] in which movements can be memorized unconsciously.

Typically, a carriage with two horses is configured so that each rein first splits up, after which each part passes through a ring on the harness of one horse and then goes onto the bit. As a consequence, if the driver's hands move in 3D space, the forces involved can be a combination of both longitudinal (along the axis of the carriage) and lateral forces and therefore influence the horses. For our purposes of using the simulator as a learning tool, we considered that the driver's hands would move along only the longitudinal axis. From the perspective of the horse and the exerted force directions, all the relevant forces during the handling of the reins can thus be considered unidirectional, along the axis of the carriage.

The concept of the simulator we developed is based on active haptic feedback. We took inspiration from the SPIDAR$\mathrm{G}$ device [15] by using a motor per rein that simulates the behavior of the horse, which is pertinent as the force exerted by the driver is supposedly unidirectional. Moreover, this principle allows to be easily adaptable to a carriage with one, two or four horses, where appropriate, depending on the desired usage. Furthermore, using such a device allows for large movement amplitudes (up to one meter), as in reality. 


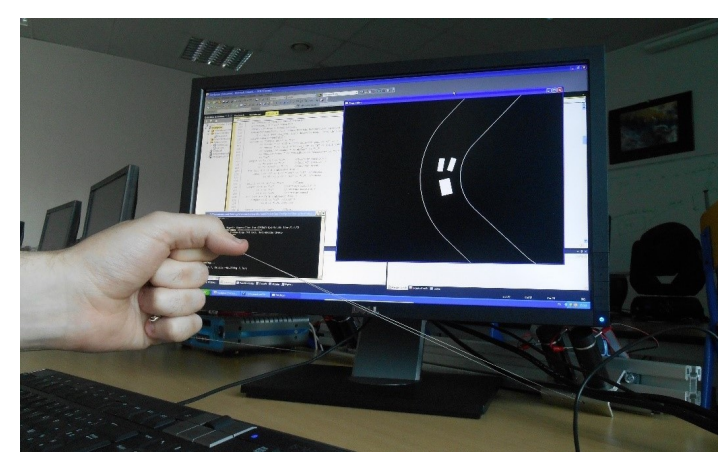

Fig. 1. First developed prototype.

The main work to perform lies in establishing laws of horse behavior that depend on the forces exerted by the driver on the reins, to in turn deduce the forces to be produced by the motors. The validation of the simulator must be done for several levels of skills in horse-drawn carriage driving to cover most of the possible usages. A parallel phase consists of studying a real carriage to obtain values that are as accurate as possible to calibrate the simulator, especially in terms of forces.

Regarding visual feedback, we developed virtual environments on OpenGL for the first prototype and then used Unity3D to obtain more realistic rendering with the final version of the simulator. Here, visual feedback is implemented through a simple screen (such as a large screen) and not in an immersive manner through head-mounted displays. Using immersive setups such as head-mounted displays can cause motion sickness [16] and introduce technical issues linked to the accurate tracking of the driver's hands and reins, which do not consider in the frame of this work.

\section{FiRst PROTOTYPE}

The first prototype was developed as a basis to validate the simulation tool to facilitate learning movements (Fig. 1).

\section{A. Haptic Interface}

The hardware interface of the simulator is derived from the SPIDAR-G device [15] within our laboratory. This device works based on the tension of wires linked to motors to produce forces. Wires are linked to a haptic probe on which forces are exerted. In the SPIDAR device we consider here, the motors are Maxon RE-max 301652 (Maxon Motor, Switzerland) DC motors equipped with 512-point per revolution quadratic encoders. The maximum forces possible to exert are $20 \mathrm{~N}$. The associated control unit integrates a processing chip that works at $200 \mathrm{MHz}$ (Hitachi SH4).

The classical configuration of the SPIDAR is a parallelepiped space in which a motor is placed at each corner, and the entire system controls a haptic point (Fig. 2 left). This arrangement is not adapted to our needs as the efforts applied to a specific position are exerted along arbitrary directions in the 3D space. Thus, we modified the typical configuration of the SPIDAR by arranging the motors so that the applied efforts are uniaxial and unidirectional toward the user (Fig. 2 right).

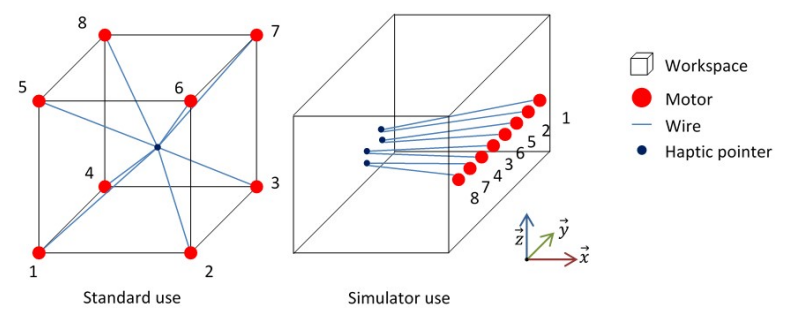

Fig. 2. Left: Classical configuration of the SPIDAR. Right: Modified configuration for simulator use.

To control all the motors, we used the libraries provided with the SPIDAR; however, these libraries do not allow for the direct control of the motors. The device is indeed controlled depending on the position and orientation of the haptic probe, and the forces produced by the motors are calculated based on the position, orientation, and the force and torque to be applied to the haptic probe. These forces are automatically distributed to the motors that apply the resulting forces in the proper direction. In the configuration of Fig. 2 left, each time a force is required along the $\vec{x}$ direction in the global frame, the system distributes it on the motors that can apply forces along this direction. This can lead to the activation of all motors, which makes it difficult to know exactly which motor applies how much force. Yet, for horse-drawn carriage simulation, we do not have a unique position, but rather two or four positions, depending on the usage of the simulator with two or four reins (one or more virtual horses). For practical reasons, we chose to group the motors in pairs, no matter the usage, which allows to obtain higher forces. For this prototype, we will consider usage with two virtual horses and up to four reins.

For the simulator's need, it is not mandatory to control the eight motors independently from one another but only four groups of motors. For this purpose, we define a virtual workspace that will be then interpreted by the SPIDAR and contain a virtual haptic probe whose position can be determined in this space. Within this workspace, we arrange the motors in a different configuration than in the classical one. The motors are positioned so that a group of motors cannot interact with one another in terms of virtual force or torque direction (Table I and Fig. 3). We devised two pairs of motors (corresponding to the use of one pair of reins) for virtual forces and two other pairs of motors (if a second pair of reins is used) for virtual torques, to distinguish the groups from each other in this virtual space. To further ensure independent control of each group, we consider the virtual haptic probe as stationary, which implies that variations in wire length against the initial lengths and the rotation of the haptic probe can be neglected. The virtual workspace is therefore defined to be of sufficiently large dimension; a dimension of $1000 \mathrm{~m}$ is arbitrarily selected. To apply virtual torques on the virtual haptic probe, the points to which the corresponding wires are attached are shifted from the original position. Motors of groups 5-6 and 7-8 are positioned in opposite directions to obtain a null net virtual force at the virtual haptic probe.

According to the positions presented in Table I, the virtual forces and torques to be applied to the virtual haptic probe are 
TABLE I

VIRTUAL COORDINATES OF THE MOTORS

\begin{tabular}{|c|c|c|c|}
\hline Motor & $\begin{array}{l}\text { Coordinates } \\
\text { in the virtual } \\
\text { space }(x, y \text {, } \\
z) \text { in meters }\end{array}$ & $\begin{array}{l}\text { Attachment point } \\
\text { coordinates on the } \\
\text { haptic probe }(x, \\
y, z) \text { in meters }\end{array}$ & Effect \\
\hline 1,2 & $(1000,0,0)$ & $(0,0,0)$ & $\begin{array}{l}\text { Force } F_{x}(\text { left rein pair } \\
1=\text { rein } 1)\end{array}$ \\
\hline 3,4 & $(0,1000,0)$ & $(0,0,0)$ & $\begin{array}{l}\text { Force } F_{y} \text { (right rein } \\
\text { pair } 1=\text { rein } 2 \text { ) }\end{array}$ \\
\hline 5 & $(0,1000,-1)$ & $(0,0,-1)$ & $\begin{array}{l}\text { Torque } T_{x} \text { (left rein } \\
\text { pair } 2=\text { rein } 3 \text {, if used) }\end{array}$ \\
\hline 6 & $(0,-1000,1)$ & $(0,0,1)$ & $\begin{array}{l}\text { Torque } T_{x} \text { (left rein } \\
\text { pair } 2 \text {, if used) }\end{array}$ \\
\hline 7 & $(1000,0,1)$ & $(0,0,1)$ & $\begin{array}{l}\text { Torque } T_{y} \text { (right rein } \\
\text { pair } 2=\text { rein } 4 \text {, if used) }\end{array}$ \\
\hline 8 & $(-1000,0,-1)$ & $(0,0,-1)$ & $\begin{array}{l}\text { Torque } T_{y} \text { (right rein } \\
\text { pair } 2 \text {, if used) }\end{array}$ \\
\hline
\end{tabular}

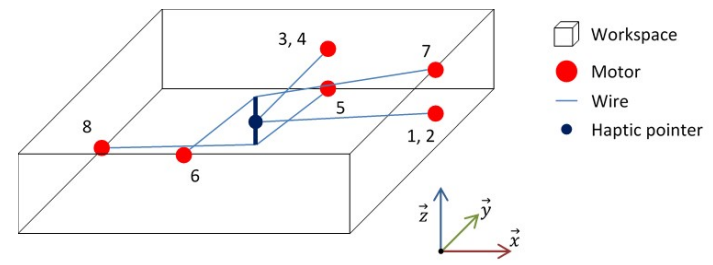

Fig. 3. Virtual workspace created for the haptic device.

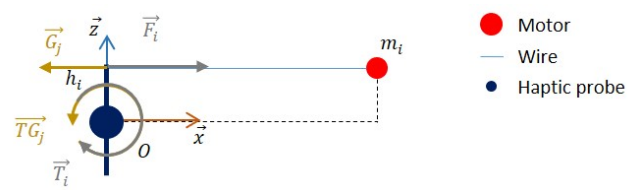

Fig. 4. Different variables of the virtual space.

computed based on the forces to be applied on each rein. Let us consider the following variables represented in Fig. 4:

- $O$ : origin of the global virtual frame

- $m_{i}$ : position of motor $i$ in the virtual workspace

- $h_{i}$ : position of the attachment point of the wire corresponding to motor $i$ on the haptic probe

- $\overrightarrow{F_{i}}$ : force exerted by motor $i$ in the global virtual frame and $F_{i}$, its norm

- $\vec{T}_{i}$ : torque exerted by motor $i$ at the center of the haptic probe in the global virtual frame and $T_{i}$, its norm

- $\overrightarrow{G_{j}}$ : effort exerted by driver pulling through rein $j$ in the global virtual frame and $G_{j}$, its norm

- $\overrightarrow{T G_{j}}$ : torque caused by the effort exerted by driver pulling through rein $j$ at the center of the haptic probe in the global virtual frame and $T G_{j}$, its norm

With the elements of Table I, it is possible to show for the left rein of pair 1 (rein 1, group 1-2) and the right rein of pair 1 (rein 2, group 3-4), respectively, that

$$
\left\{\begin{array}{l}
\overrightarrow{G_{1}}=\overrightarrow{F_{1}}+\overrightarrow{F_{2}}=G_{1} \vec{x} \\
\overrightarrow{T G_{1}}=\overrightarrow{T_{1}}+\overrightarrow{T_{2}}=0
\end{array},\left\{\begin{array}{l}
\overrightarrow{G_{2}}=\overrightarrow{F_{3}}+\overrightarrow{F_{4}}=G_{2} \vec{y} \\
\overrightarrow{T G_{2}}=\overrightarrow{T_{3}}+\overrightarrow{T_{4}}=0
\end{array}\right.\right.
$$

Similarly, for the left rein of pair 2 (rein 3, group 5-6) and the right rein of pair 2 (rein 4, group 7-8), respectively, it is possible to show that

$$
\left\{\begin{array}{l}
\overrightarrow{G_{3}}=\overrightarrow{F_{5}}+\overrightarrow{F_{6}}=0 \\
\overrightarrow{T G_{3}}=\overrightarrow{T_{5}}+\overrightarrow{T_{6}}=\left(h_{5}+h_{6}\right) \frac{G_{3}}{2} \vec{x} \\
\overrightarrow{G_{4}}=\overrightarrow{F_{7}}+\overrightarrow{F_{8}}=0 \\
\overrightarrow{T G_{4}}=\overrightarrow{T_{7}}+\overrightarrow{T_{8}}=\left(h_{7}+h_{8}\right) \frac{G_{4}}{2} \vec{y}
\end{array},\right.
$$

As presented in Table I, the distances of the attachment points of the wires on the virtual haptic probe are equal to $1 \mathrm{~m}$ ( $\left.h_{5}=h_{6}=h_{7}=h_{8}=1\right)$ and are selected such that a 1 $\mathrm{N}$ force on the second pair of reins corresponds to a $1 \mathrm{Nm}$ torque around $\vec{x}$ and $\vec{y}$, respectively.

Thanks to this configuration, the four groups of motors are independent from one another. With the SPIDAR controlled in force and torque, the parameters to be applied to generate the desired forces on the reins are as follows:

$$
\begin{array}{r}
F_{S P I D A R}=G_{1} \vec{x}+G_{2} \vec{y} \\
T_{S P I D A R}=G_{1} \cdot(1 m) \vec{x}+G_{2} \cdot(1 m) \vec{y}
\end{array}
$$

\section{B. Behavioral Laws}

The main need for this prototype is to enable the simulation of a horse-drawn carriage at four different gaits: stop and backward motion, walk (between 6 and $8 \mathrm{~km} / \mathrm{h}$ ), slow trot (between 13 and $16 \mathrm{~km} / \mathrm{h}$ ) and fast trot (between 25 and 30 $\mathrm{km} / \mathrm{h}$ ). To simplify, random events impacting the behavior of the horses were not considered. The behavioral laws of the carriage primarily focused on the computation of the carriage speed, the forces to be applied to the reins and the rotation of the virtual horses. These laws mainly depend on the length $L$ of the reins from their initial position. In the following, we consider usage with two virtual horses and two reins.

1) Carriage speed: The simulated carriage speed is calculated based on the mean length of the wires, as a first approximation to the fact that the carriage slows when the driver pulls both reins; although, in a real situation, deceleration does not always depend on the reins' pulling length. The simulated carriage speed is then calculated as follows (Fig. 5 left):

$V=V_{\max }+\left(V_{\max }-V_{\min }\right) \frac{\overline{L_{\min }}-\bar{L}}{\overline{L_{\max }}-\overline{L_{\min }}}, V \in\left[V_{\min }, V_{\max }\right]$

where $\bar{L}$ is the mean length of the wires, $\overline{L_{\min }}$ and $\overline{L_{\max }}$ are the mean lengths of the wires corresponding to the minimum $V_{\min }$ and the maximum $V_{\max }$ speeds respectively, which are defined by the gaits mentioned above. In practice, $\overline{L_{\min }}$ is negative, which corresponds to a situation in which the driver moves the reins forward from their initial position.

2) Exerted forces: With a two-horse carriage, each rein can control both horses; however, the effect of the reins on both horses is not produced at the same moment. The forces exerted on the reins are then calculated by (Fig. 5 right)

$$
\begin{cases}F_{e}=F_{\text {min }}+\left(F_{\text {int }}-F_{\min }\right) \frac{L-L_{1}}{L_{2}-L_{1}} & \text { if } L<L_{2} \\ F_{e}=F_{\text {int }}+\left(F_{\max }-F_{\text {int }}\right) \frac{L-L_{2}}{L_{\max }-L_{2}} & \text { otherwise }\end{cases}
$$

where $F_{e} \in\left[F_{\min }, F_{\max }\right] ; F_{\min }, F_{\text {int }}, F_{\max }$ are the minimum, intermediate and maximum applied efforts, respectively; $L_{1}$ and $L_{2}$ are the lengths from which the rein affects the first 

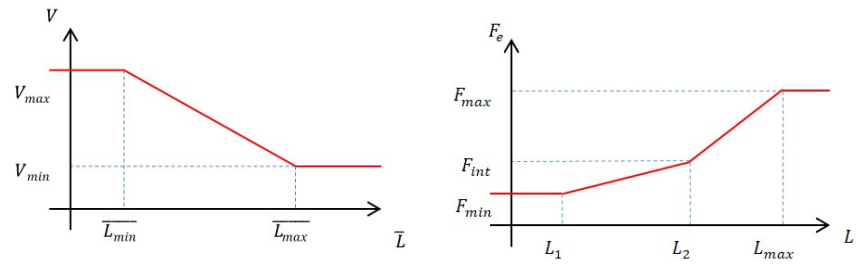

Fig. 5. Left: carriage speed law. Right: exerted forces law.

TABLE II

VALUES OF THE PARAMETERS IN $F_{s}$

\begin{tabular}{|l|l|l|l|}
\hline & Walk & Slow trot & Fast trot \\
\hline$A_{F_{s}}(\mathrm{~N})$ & 4.5 & 5.5 & 6.5 \\
\hline$f_{F_{s}}(\mathrm{~Hz})$ & 1.5 & 1.75 & 2.0 \\
\hline
\end{tabular}

and second horses respectively; and $L_{\max }$ is the length from which the rein does not have any effect. Here $F_{\max }$ is equal to $40 \mathrm{~N}$, which corresponds to the maximum force exerted by the paired motors.

To enhance realism, we added a force $F_{s}$ to $F_{e}$, representing the force resulting from the movement of the horse's body on the reins. This movement was considered to be sinusoidal for the sake of simplicity. Thus, $F_{s}$ is also expressed as a sinusoid:

$$
F_{s}=A_{F_{s}} \sin \left(2 \pi f_{F_{s}} t\right)
$$

where $A_{F_{s}}$ is the amplitude of the swing force and $f_{F_{s}}$ is the frequency associated with this force. These parameters are tunable depending on the characteristics of the horse to be simulated and its gait. For the simulator, we set the values reported in Table II.

3) Horse orientation: To orient the horses and thus simulate rotational displacements, we chose to calculate the rotation angles from the difference of wire lengths. This allowed to avoid the use of the absolute position of the reins in the virtual workspace. Similarly, we chose to not rotate both horses at the same moment or with the same amplitude to simulate the fact that horses should not collide with one another while turning. The horse orientation is calculated as follows (Fig. 6):

$$
\begin{aligned}
& \begin{cases}\alpha_{l}=\alpha_{\max } \frac{\Delta L-\Delta L_{1}}{\Delta L_{\max }-\Delta L_{1}} & \text { if } \Delta L>\Delta L_{1} \\
\alpha_{l}=r \cdot \alpha_{\max } \frac{\Delta L+\Delta L_{2}}{\Delta L_{\max }-\Delta L_{2}} & \text { if } \Delta L<-\Delta L_{2}\end{cases} \\
& \begin{cases}\alpha_{r}=r \cdot \alpha_{\max } \frac{\Delta L-\Delta L_{2}}{\Delta L_{\max }-\Delta L_{2}} & \text { if } \Delta L>\Delta L_{2} \\
\alpha_{r}=\alpha_{\max } \frac{\Delta L+\Delta L_{1}}{\Delta L_{\max }-\Delta L_{1}} & \text { if } \Delta L<-\Delta L_{1}\end{cases}
\end{aligned}
$$

where $\alpha_{l} \in\left[-r \cdot \alpha_{\max }, \alpha_{\max }\right]$ and $\alpha_{r} \in\left[-\alpha_{\max }, r \cdot \alpha_{\max }\right]$, $\Delta L=L_{l}-L_{r}$ is the difference of length between the left and the right reins, $\Delta L_{1}$ and $\Delta L_{2}$ are the differences of the length impacting the first and the second horses, respectively, $\Delta L_{\max }$ is the maximum difference of length, $\alpha_{\max }$ is the maximum rotation of the horses, and $r<1$ is the ratio between the right and left rotations.

The constant parameters are tunable depending on the gait and the characteristics of the horses to be simulated.

\section{Prototype Validation}

1) Simulation loop: The simulation loop comprises all the steps required to obtain a real-time simulation of the horse-

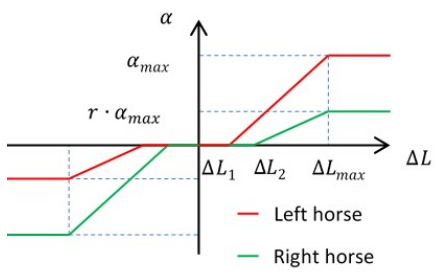

Fig. 6. Horse orientation law.

drawn carriage, consisting of four main steps. The first consists of calculating the virtual forces and torques in the virtual workspace. Though we considered the virtual haptic probe to be stationary, these calculations consider the variations of the position and orientation of the probe to be more accurate. The second step consists of updating the haptic device by applying to the motors the values calculated in the previous step; the wire length is recorded, as well. Then, the behavior of the virtual carriage is updated following the method described in the previous part. The position and orientation of the virtual carriage are then rendered in the application.

2) Simulation operation: In a real carriage, drivers can use their voice in addition to the reins to command a change of gait. To simulate this aspect, the change from a gait to a quicker one (acceleration) in the simulation is achieved orally by the trainee; a second person then presses a key on a keyboard to operate this change. Deceleration is, in contrast, achieved by pulling the reins.

To turn to the left or right, the user pulls on the corresponding rein and maintains the other rein in its current position.

3) Experiment and results: We performed a first validation test of the simulator with the help of a professional highlevel driver with more than 20 years of experience in horsedrawn carriage driving to determine the proper parameters in the behavioral laws defined above. The laws were accordingly tuned to obtain realistic sensations.

We then asked 11 participants, novices at horse-drawn carriage driving, to evaluate the simulator by following the pre-defined path depicted in Fig. 7, considering a carriage with two horses. The path incorporated straight lines and left and right turns with different steering radii. The rendering of the path and the carriage was very basic (achieved with OpenGL); however the main goal here was to observe the movements performed by the participants without evaluating the influence of the visual rendering on performance. Each participant was asked to maneuver one round of the track, during which several variables were recorded: the forces exerted in the reins, the achieved trajectory (measured at the center of the carriage), the time to complete the round, and the mean speed.

Figure 8 depicts an example of the forces exerted and trajectory realized by one subject. We can see that the smaller the steering radius, the higher the exerted forces, which is in accordance with the implemented behavioral laws. The performance results of all participants are presented in Fig. 9. Here, we can notice strong differences in terms of mean forces exerted between the participants; for example, participants 1 and 7 drove on average at the same speed and took the same 


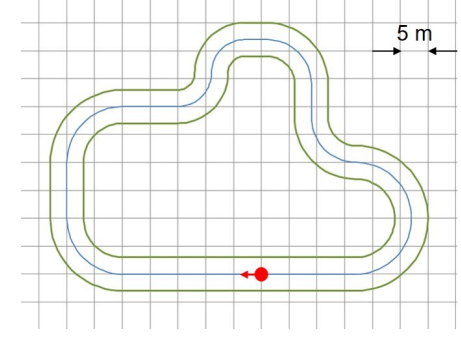

Fig. 7. Test track.

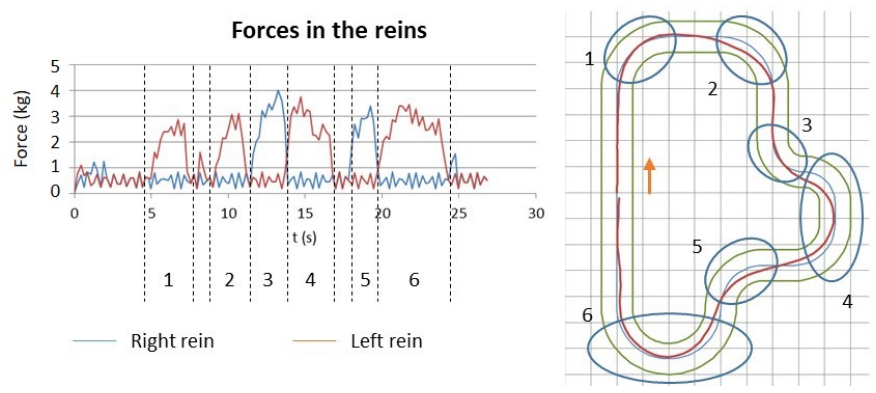

Fig. 8. Forces exerted by one participant with the associated realized trajectory. Numbers in the graph (from 1 to 6) refer to the ellipses marked on the track.

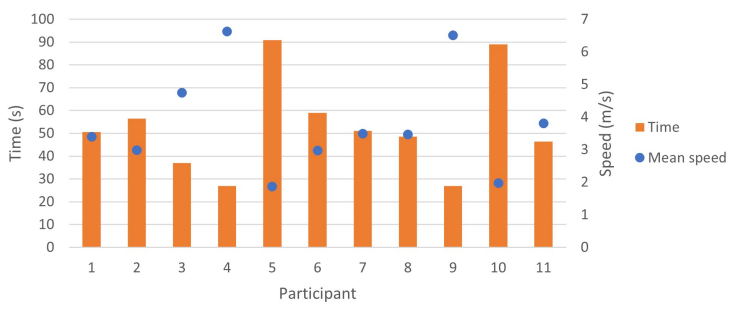

(a) Track completion time and mean speed

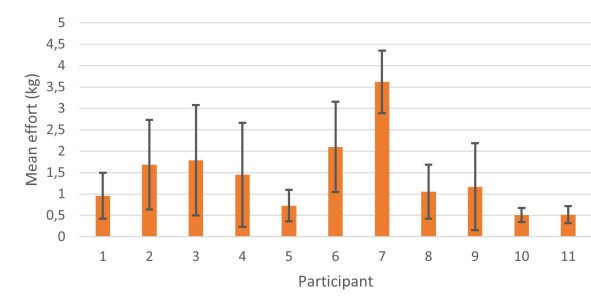

(b) Mean forces and standard deviation

Fig. 9. Experimental results.

amount of time to complete the track, but participant 7 exerted forces about four times higher than participant 1 . Overall, we observed that the subjects could handle the simulator quickly despite nobody having prior experience with driving horsedrawn carriages. Furthermore, we noted that only two subjects drove off the path during the rounds; such instances occurred only in short-radius turns.

An interview was performed with the participants at the end of the round. The participants indicated being conscious of the horses' movements thanks to the sinusoidal forces exerted on the reins. However, speed control appears to have been forgotten by most of the subjects during the tests. The participants primarily maintained the same gait all along the

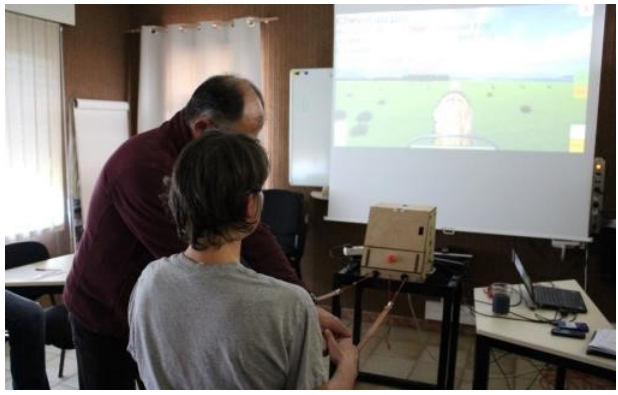

Fig. 10. coMtactS prototype in operation during a training session.

track, which explains why some drove off the path.

\section{Discussion}

The first results reveal the interest of training future drivers on a simulator before learning with real horses, as users had different driving strategies that could be harmful to real horses. The simulator was validated by a professional driver and indicated to be easy and fast to handle, even for users who were novices in carriage driving.

Nevertheless, several improvements must be considered. On one hand, the maximum forces provided by the motors cannot exceed $20 \mathrm{~N}$ each; however, as mentioned above, real efforts are estimated to reach $100 \mathrm{~N}$ or greater while cantering. Therefore this first simulator is not suitable for real training. On the other hand, the behaviors of the virtual horses as described here are basic. One specificity of horsedrawn carriage driving is the existence of constant and subtle communication between the horses and the driver through the reins, which is not reproduced here. Especially in real situations, forces can be exerted on the reins by either the horse or the driver. Our first prototype does not include the ability to distinguish the origins of the forces. Furthermore, the visual rendering is very simple. The object of this simulator is to place learners in a position and viewpoint that match real situations.

\section{Second Prototype: coMtacts Simulator}

To address the points raised regarding the first prototype, we designed a second prototype, called coMtactS (Fig. 10). Since it aimed for a better understanding of contact, unlike the first prototype, we decided to restrict it to a singlehorse carriage. The first step consisted of precisely quantifying the real forces that occur between the horse and the driver. Afterward, to improve the accuracy of the simulator, we performed ethnographic and self-confrontation interviews with drivers based on real horse-drawn carriage activities. Then, we developed the simulator itself and the hardware and software, accordingly.

\section{A. Real Data Collection}

To quantify the efforts that occur between the horse and the driver, we attached an IPOS (ex-Centaur) rein sensor (IPOS, Netherlands) to the reins of one horse (Fig. 11). This sensor is specifically designed for the equestrian field and is composed 


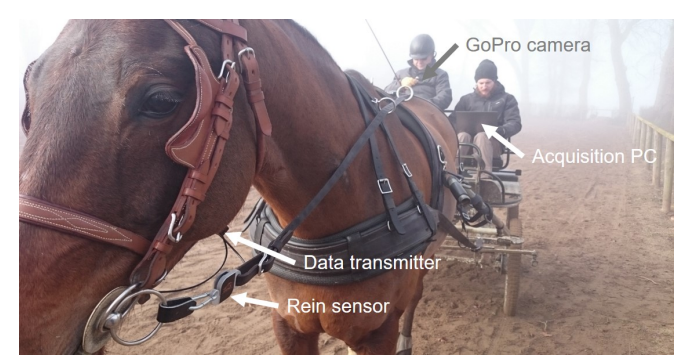

Fig. 11. Setup for the data collection with the rein sensor attached to the reins.

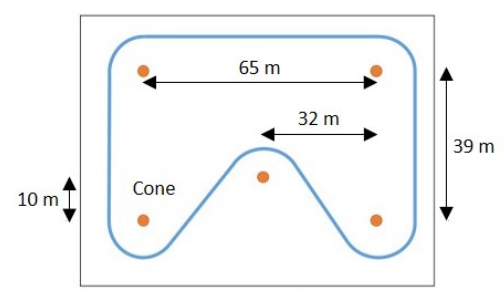

Fig. 12. Path followed by the drivers during the data collection.

of two modules attached to the left and the right reins. Forces are collected at $80 \mathrm{~Hz}$ and data are transmitted by a module attached to the harness to a PC via Bluetooth. To simplify the data collection, we considered a carriage with only one horse.

The data collection was conducted as follows:

- As each horse has unique behavior, four different horses were selected to obtain an overview of the main behaviors that are encountered. These horses had different characteristics in terms of training, behavior, and balance.

- Two professional high-level drivers with different driving strategies were asked to drive each horse.

- The drivers had to drive along the pre-defined path illustrated in Fig. 12. The path was on a dirt floor field and consisted of straight lines and left and right turns. Cones were placed at each corner of the path to indicate the locations of turns for the drivers. For each horse, the drivers needed to perform three rounds with a trotting gait clockwise followed by three rounds with a trotting gait counterclockwise. The trotting gait was chosen because it represents the gait for which forces should be the most identifiable.

- A GoPro camera was attached to the torso of the drivers to enable viewing their actions on the reins and for allowing matching between the measured forces and the actions taken by the drivers. As the camera is equipped with a microphone, the drivers' voice commands were recorded, as well. The synchronization between the forces and the drivers' view allows to better distinguish whether it is the driver or the horse that exerts the forces.

Figure 13 presents the forces exerted on the left and right reins for two different horses with the same driver in each round. We included images captured from the GoPro camera to highlight key moments on the graphs. We can see that each horse has a different behavior, resulting in different evolutions of forces. Moreover, we can observe that for one horse, each round presents different evolutions of forces, as well. This phenomenon was observed no matter which horse or driver. Several reasons explain such differences: Each horse is naturally different, the behavior of the horse and the driver's reactions differ each time, and more or less important unbalance of the horse occurs at each time. Therefore, even with the video synchronization, it is difficult to deduce strongly salient characteristics for the forces. Nonetheless, we could understand that (i) the moments when the forces were high corresponded to moments when the drivers exerted forces (e.g., to turn or slow down), (ii) the moments when forces from both sides were asymmetric corresponded to moments when turns were performed, the drivers asked the horse to go straight again, or the drivers attempted to rebalance the horse. In Fig. 13a, we can remark the presence of force peaks of around $12 \mathrm{~kg}$, corresponding to moments when the horse sped up. The driver pulled on both reins to slow down and maintain a trotting gait, which results in a sudden decrease of the forces to recover the contact (seen in Fig. 13a, left, at around $40 \mathrm{~s}$ ). Nonetheless, based on these measures, we observed that overall, no matter which horse or driver, forces did not exceed $8 \mathrm{~kg}$ on average for a trotting gait.

\section{B. Interviews with Drivers}

Parallel to the real data collection, self-confrontation interviews with drivers were conducted following Theureau's procedure [17] to understand how they drive horse-drawn carriages and what they focus on while driving. Through questions on what the drivers do, these interviews allowed for measuring drivers' activities; here, an activity was defined as the dynamics of asymmetrical interactions between an actor and his or her environment.

Four expert drivers (three men) were asked to drive a singlehorse carriage at different gaits on a semi-constrained track including turns, different terrains and slight slopes. Prior to driving the carriage, the drivers first needed to scout the track via bike for 20 minutes so that they could plan the actions to take with the carriage. While driving the carriage, videos were captured from the drivers' viewpoint using a GoPro camera. After the driving session, the drivers were invited to view the videos and comment on them as much as possible.

According to the interviews, the fundamental concerns of drivers and their crucial intentions are, in particular, the safety and maneuverability of the carriage and the physical and mental integrity of the horse. For this, they seek the following:

- To establish and maintain virtuous communication between the driver and the horse via the reins. This is achieved a) by interpreting and acting, according to the situation, on the levels and types of forces or their timings and durations (correlation of the horse/environment, driver/environment interaction) and b) by using other cues such as the voice, the whip, the carriage brake and the vibrations of the seat.

- To optimize the dynamic system horse/harness/carriage by minimizing possible disturbances of the harness and/or carriage on the horse's locomotion. While moving, the drivers aim to achieve a "continuity of traction".

- To guarantee the availability of the horse to the commands and the quality of the route produced (which 

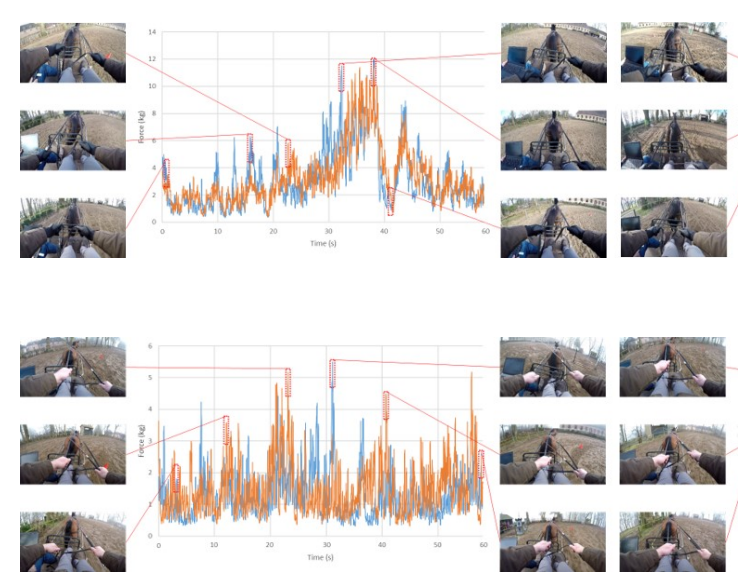

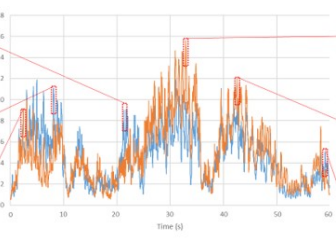

(a) For one horse
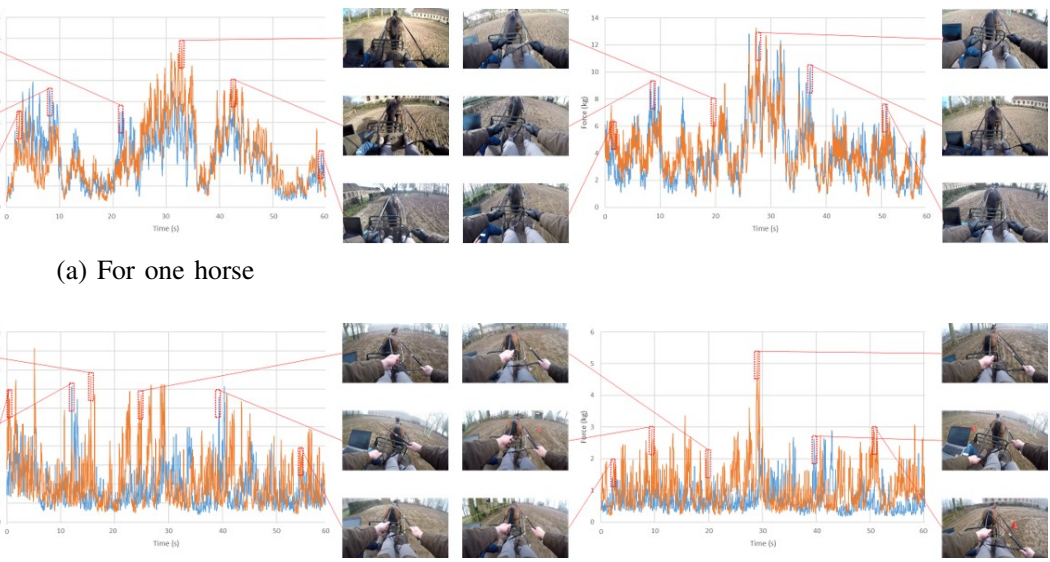

(b) For a different horse

Fig. 13. Force data for two horses with the same driver in each round in the clockwise direction. In blue, left rein; in orange, right rein.

often consists of turns) by building and supporting its equilibrium and forward motion.

- To guarantee the physical integrity of the horse by paying particular attention to its locomotion and the functioning of its muscle chains.

\section{Haptic Interface}

The haptic interface follows the same principle as the first prototype: Wires simulate the reins and are driven by motors to produce forces. As the collected data revealed that forces can reach up to $8 \mathrm{~kg}$, the hardware used in the first prototype was not as powerful as expected; therefore, we decided to develop an interface from scratch.

For this new prototype, the motors used are Doga 168.4111.30.04 (Doga, Spain) DC motors, which have a 16 pulse per round resolution. Furthermore, the nominal torque is $0.75 \mathrm{Nm}$. Two motors were installed, one for each rein.

To distinguish the origins of the forces, from either the horse or the driver, we added to each motor a $10 k \Omega 10$ turn wirewound potentiometer (Vishay, USA) that has linear precision to the millimeter. The potentiometer acts as an encoder: When the variation of the voltage as measured at the potentiometer is negative, we consider the force to originate from the horse, but when the variation of voltage is positive, the force originates from the driver. For practical reasons, we converted the measured variation of voltage to a variation of the length of the wires $\Delta L$. The force exerted by the user is then deduced based on the position of the rein with respect to its position in the previous simulation frame.

The force applied by the motors is then:

$$
F=F_{\text {driver }}+F_{\text {horse }}
$$

where $F_{\text {driver }}=\alpha \Delta L$, and $\alpha$ is a coefficient to convert the measured voltage to a length that depends on the diameter of the pulley attached to the potentiometer. $F_{\text {horse }}$ is detailed in the next section.

The system is controlled by two Arduino UNO boards, one used to command the motors and the other used to receive values from the potentiometers. We split the control into two boards to avoid any latency in the system.

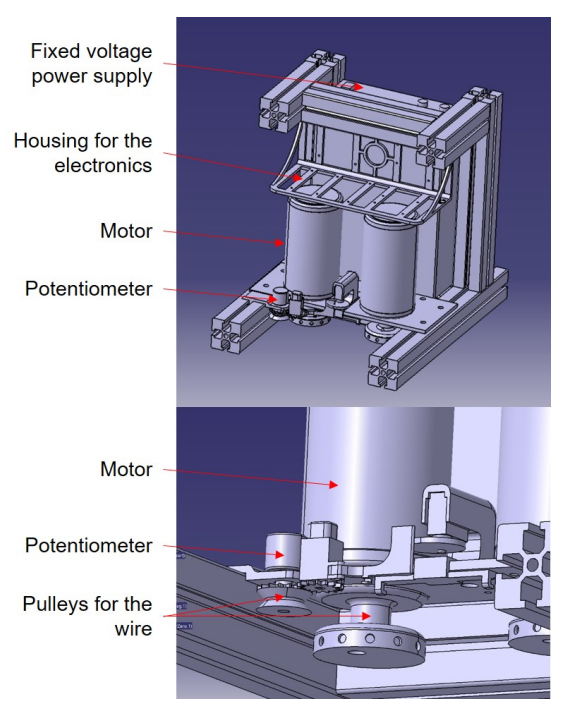

Fig. 14. Design of the second prototype, with a focus on the motor+potentiometer system (bottom).

The design of the system is presented in Fig. 14. The final prototype was produced using 3D printers for small parts, such as pulleys, and laser cutting for the casing (Fig. 15).

\section{Simulator Functionalities}

Based on the real data collection and the self-confrontation interviews with the drivers, the core of the simulation software associated with the hardware implements two distinct concepts:

- interpretation of the forces produced by the driver

- horse's behavior regarding the commands given by the driver

1) Interpretation of the forces: For each rein, the software determines the command issued by the user. This command can take five different states:

- Give in: The driver gives the rein some slack. This means that he or she does not hold the rein tightly (exerted force less than $0.4 \mathrm{~kg}$ ) or that the length of the unwound wire is low (less than $20 \mathrm{~cm}$ ). 


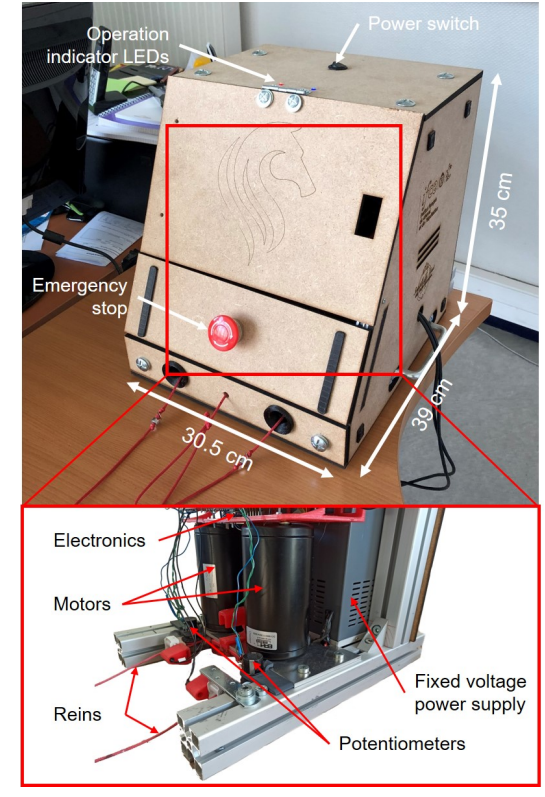

Fig. 15. Final prototype.

- Contact: A minimum force is applied to the rein. This force is configurable; by default, the contact zone is between 0.4 and $1.4 \mathrm{~kg}$.

- Maintain: When the horse exerts a force, the opposite force is applied.

- Pull: The driver applies a force higher than the contact zone (higher than $1.4 \mathrm{~kg}$ ) plus possibly the horse's force.

- Act: The driver pulls higher than the contact zone (higher than $1.4 \mathrm{~kg}$ ) plus possibly the horse's force for a period between 0.25 and 3 seconds (e.g., to turn).

2) Horse behavior: The horse being a non-deterministic system, we developed a behavioral engine that could reproduce the most encountered behaviors of horses. This engine is based on a state machine system, and the horse can be in one state at any given time. Depending on the commands it receives, it will either remain in its current state or change it. According to this state, the speed, animation (visual and sound), and force feedback will be computed. Seven states were defined according to professional drivers:

- Rest: The horse does not move.

- Walking: The horse moves forward at a constant gait (around $7 \mathrm{~km} / \mathrm{h}$ ).

- Walk to the right: The horse turns to the right while walking.

- Walk to the left: The horse turns to the left while walking.

- Trot: The horse trots straight ahead at a constant gait (around $14 \mathrm{~km} / \mathrm{h}$ ).

- Trot to the right: The horse turns to the right while trotting.

- Trot to the left: The horse turns to the left while trotting.

The rules for state changes were defined as follows:

- To accelerate (from rest to walk, or from walk to trot), reins must be given in for 0.5 seconds.

- To turn, the driver must act on the inner rein (the rein on the turning direction's side) and maintain contact on the

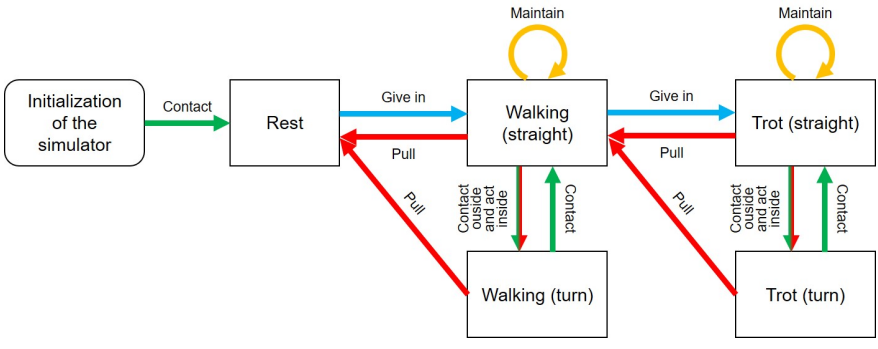

Fig. 16. State change diagram.

external rein (the rein on the turning direction's opposite side). The intensity of the turn depends on the intensity of the external contact.

- To decelerate (from trot to walk or from walk to rest), the driver must pull on both reins.

- After acceleration or deceleration, contact must be reestablished on both reins for 0.5 seconds before the horse accepts new orders.

In addition to these baseline rules, the following rules were added to manage driving errors:

- If the driver acts on one rein but gives in on the other rein, the horse will turn in the wrong direction. This may appear counter-intuitive, but this is a natural reaction.

- If during a turn the driver pulls more than he or she acts, the horse decelerates.

The diagram with the different state changes is depicted in Fig. 16. Each arrow represents an action on both reins.

In addition to the behaviors implemented above, we added to the horse's behavior a virtual state of mind (or "mood") that depends on the personality determined by the trainer at the beginning of the simulation. The virtual horse will tend to give headbutts regularly, displayed by a force on the opposite rein to the side of the headbutt. The state of mind will vary during the simulation on a gauge. This gauge fills up over time if the driver commits driving errors, such as if he or she pulls for too long on the reins or if, in contrast, he or she gives in for too long. Otherwise, the gauge decreases over time if no errors are made. If the horse's mood exceeds a defined threshold, headbutts will be triggered with a strength and frequency that depend on the horse's personality and will.

The total force exerted by the horse can then be written as follows:

$$
F_{\text {horse }}=F_{\text {gait }}+F_{\text {mood }}
$$

where

$$
\begin{aligned}
& F_{\text {gait }}=A_{p} e^{-\left(6 t_{p}-3\right)^{2}}, t_{p}=\frac{t}{T_{p}}-\left\lfloor\frac{t}{T_{p}}\right\rfloor \\
& F_{\text {mood }}=A_{m} \sum_{k} \Lambda\left(2 t-\left(2 T_{m} k+1\right)\right)
\end{aligned}
$$

$F_{\text {gait }}$ is the natural force from the horse's head, whose amplitude $A_{p}$ and period $T_{p}$ change according to the gait, and is represented as a Gaussian to simulate the swing effect of the horse head; $F_{\text {mood }}$ is the force representing the horse's mood, whose amplitude $A_{m}$ and period $T_{m}$ change according to the personality set at the beginning of the simulation and the evolution of the mood gauge, and is represented as a triangular function $\Lambda$ to simulate a quick headbutt; and $t$ denotes the 
simulation time. The different constant values were tuned by trial and error to obtain similar behaviors as observed from the real data collection; professional drivers provided finetuning to achieve sensations similar to those that exist with real horses.

3) User interface: The user interface was implemented under Unity3D, and all the software components associated with the communication with the hardware were written in C\#.

The simulator software integrates four play modes:

- Replay mode: In this mode, real forces data from the real data collection are read and sent to the hardware. The driver is thus in a passive mode, merely feeling the forces; these forces are synchronized with a view from a GoPro camera. Comments provided by the real driver supplement the experience. This mode is intended to obtain an initial understanding of the forces involved in a real carriage driving situation.

- Free driving mode: This mode is the main simulator part and allows for a driver to train on a virtual horse-drawn carriage with the haptic feedback described above. The viewpoint is set as in reality, behind the horse. The virtual environment consists of a large field with few obstacles such as trees.

- Training mode: This mode allows drivers to train on a predefined virtual track.

- Time-limited mode: This mode is similar to the training mode, except that a timer measures the time taken by the driver to complete the track and allows to evaluate the driver.

The user interface of the virtual environment is presented in Fig. 17. On each side of the screen, information is provided to the driver corresponding to each rein. A white square denotes the force exerted by the driver, while a colored square denotes the state of command, as described in Section IV-D1. The color changes according to the state: light blue for the give-in state, green for the contact state, yellow for the maintain state, and red for the pull and act states. As the goal is to assimilate the notion of contact, the driver must have the square on each side remain green for as long as possible. A timer displays the time the driver remains in the corresponding state. We thus suppose that providing such a cue to the driver in addition to the force feedback will allow to memorize the proper movements, based on the principle of procedural memory [14]. Furthermore, on the right side of the interface, an additional colored square indicates the value of the mood gauge; its color changes according to the mood.

Before the simulation starts, the trainer can parameterize the virtual horse in terms of personality. Values set based on professional drivers' experience can be selected (easy-, intermediate-, or hard-to-manage horse). Moreover, the walking and trot speeds can be defined among proposed values. In addition, a contact zone can be set corresponding to the minimum and maximum forces exerted by the driver for which contact is achieved.

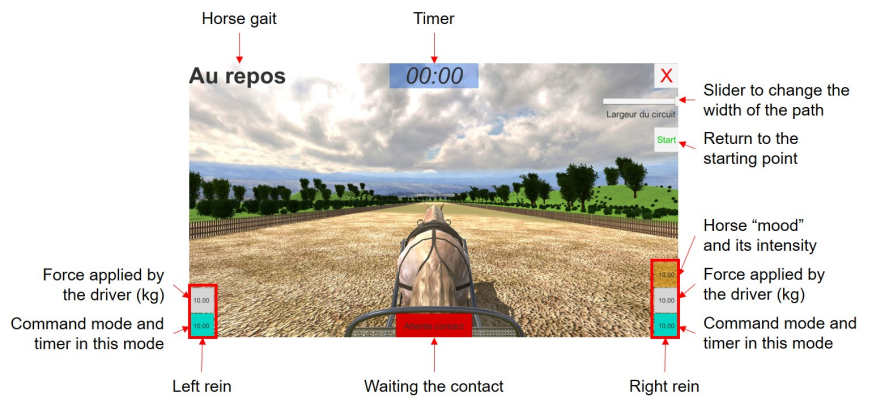

Fig. 17. User interface.

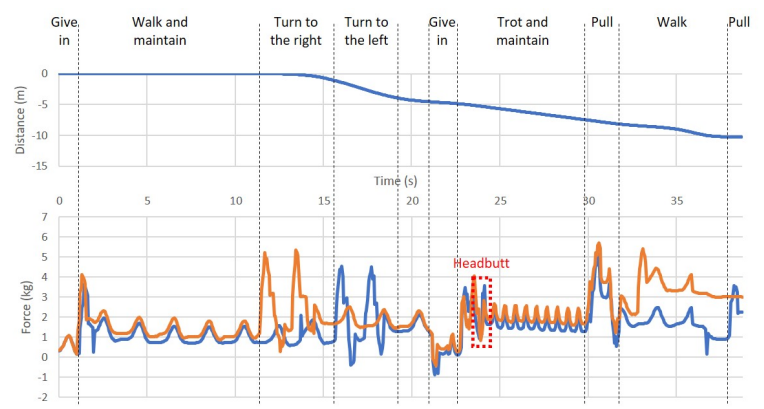

Fig. 18. Force data during a free virtual driving session, with the associated behaviors and events. In blue, left rein; in orange, right rein. The driven path is depicted on the top.

\section{E. Validation}

Figure 18 presents the forces exerted on the left and right reins during a free virtual driving session on a flat field with an easy-to-manage horse. The different behaviors of the virtual horse, as implemented in Section IV-D, can be clearly seen, including headbutts occurring depending on the horse's mood.

The prototype was further evaluated with six trainees under the supervision of two trainers. These trainees already had four months of experience in horse-drawn carriage driving. Emphasis was placed on the learners' feedback rather than on a comparison between the simulated and real force values. Indeed, based on the practice of horse-drawn carriage driving, the most important aspect to assess is behaviors and sensations [3]. Additionally, an absolute assessment is nearly impossible to achieve as horses are non-deterministic systems; thus, simulated and real forces can never be the same, even if a real carriage is fully instrumented.

The trainees were asked to experiment with the simulator in replay mode, free driving mode, and training mode for a total time of one hour and twenty minutes. They were assisted by the trainers who provided advice while driving. Interviews were then organized for about 20 minutes to obtain comments on the use of the simulator.

Based on the interviews, we observed that the trainees were aware that what is learned during the simulation can potentially be applied in real situations. Statements such as "The simulator will allow me to test things to find the right handling when back with my horse" were made.

Furthermore, the trainees reported that the first benefit of the simulator is its playful and interactive aspect. Indeed, one of 
the noteworthy elements of the trainees' feedback is the lack of attractiveness and the boredom associated with the classical teaching methods (e.g., the traditional weight simulator) due to its persistently identical environment: "It is going to be more playful with this simulator because I am bored with the weight simulator."

The simulation experience with the coMtactS prototype was positive when the trainees reported their kinesthetic and visual perceptions experienced from using the simulator; examples include "We have real sensations of driving" and "You still feel like it tells you what you are doing." In addition, the simulator allows the trainees to take time to search for solutions via trial and error: "This is the kind of tool that allows me to practice until it works."

Another interesting observation is that the oral exchanges between the trainer and trainee improved thanks to the coMtactS prototype, as technical points could be addressed in a more peaceful and in-depth manner than in a real situation. The conditions of the simulated situation made it easier for the trainees to verbalize their practices or difficulties and highlight force asymmetries between the left and right hands, thanks to the indicators present in the user interface.

\section{F. Discussion}

Based on the use of the simulator by the trainees, we noted two distinct behaviors: a first behavior in which trainees struggle to interact with the interface by opposing it to real driving situations (we had one case), and a second in which trainees accept how the simulator works, allowing to explore a new relationship with the virtual horse. The first behavior in front of the simulator establishes a distance between reality and simulation that cannot be reduced; this distance prevents the trainee from entering a playing situation that could have development potential. Trainers have an important role to play in explaining how the simulator works and its limits. Trainees and trainers must be closely complementary in their exchanges, as they are both convinced that it can provide interesting insights into their work and provide strong improvements in the practice of horse-drawn carriage driving.

Nevertheless, feedback from the trainees was positive as the sensations experienced during the simulation and the behaviors of the virtual horse were close to being realistic. As mentioned above, we focused on a purely subjective assessment, as the horse-drawn carriage field is strongly based on kinesthetic perceptions and subjective sensations. The question of the validity of simulators is a well-known issue that is often discussed, especially in the driving simulation field [18]. In our case, as demonstrated in the data collection, even if a driving session is repeated on the same track and with the same horse, driver, and conditions, the behaviors always change due to the non-deterministic nature of the horse; therefore, each driving experience cannot be reproducible exactly. As a result, the absolute validity of our simulator is not relevant and only relative validity could be achieved. It is worth noting that we did not perform a comparative test between with- and withoutsimulator trainees, since the approach adopted for this study consisted of responding to an expectation of the trainees in terms of practice and interaction between the trainer and the trainees; however, this will be considered in future work.

\section{CONCLUSiON}

In this paper, we presented two prototypes developed for the realization of a horse-drawn carriage simulator. Both the hardware and the software are explained. The first prototype was designed to ensure that such a tool could be relevant in facilitating learning gestures. A second prototype was then created to address issues raised by the first, with a focus on the notion of contact. The simulator is easy to install as it simply requires to be plugged in via standard USB ports. The core of the interface consists of motors exerting forces on wires and potentiometers to distinguish between the forces exerted by the drivers and those exerted by the virtual horse. The device provides force feedback whose level is coherent with that encountered in real situations. In addition, the most encountered behaviors of the horses were integrated to enhance realism. The image is displayed to the trainees as if they were driving a real carriage. Moreover, several play modes were implemented to scale the learning progression. The coMtactS simulator was used in real training sessions and the feedback from the trainees exhibited positive outcomes as they could sense realistic forces and reactions.

The coMtactS prototype was designed with a particular focus on movements and the feeling of subtle forces. However, to be fully realistic, the carriage simulator must consider several aspects. First, we considered that the drivers' hands were moving only along the axis of the carriage, whereas they can move also to the left or right. We will work on implementing this possibility in the simulator. Second, other sensory cues frequently used by drivers will be added; for example, the voice is complementary to controlling the horse, and drivers also rely on the vibrations felt through the seat to guide the carriage. Third, we did not offer the ability to tune the simulator to train students to learn to use minimal rein tension to ask for desired behaviors from the horse. This functionality will be added as an important feature to help to prevent injuries on horses. Fourth, we will consider refining the mood-related behaviors added to the simulation to address for instance the impacts of the surroundings. In parallel, work has already been conducted to integrate this simulator into a global renovated teaching-learning system for horse-drawn carriage driving that includes in particular a video platform called Ercam. Based on past work on the analysis of video training to support professional development [19], assets, limits and conditions were identified to use video as a complement to the coMtactS simulator and field driving situations [3]. Last, the principle developed for this simulator could be applied to sports fields other than equestrian driving.

\section{ACKNOWLEDGMENT}

The authors would like to thank Louis Basty, Renaud Vinck and Michel Héreau who are professional horse-drawn carriage drivers for their precious advice during the development of the simulator, as well as Mathieu Tournadre, Louis Jeannin, Nicolas Caligiuri and Jean-Claude Da Silva for their help in the development of the prototypes. 


\section{REFERENCES}

[1] S. Leblanc and G. Azéma, "Transition au sein de l'école française du cheval attelée. Expérience d'un développement professionnel médié par la recherche." in Se développer comme professionnel dans les occupations adressées à autrui : Conditions, étapes et modalités dans une réalité du travail sous pression., J. Mukamurera, J.-F. Desbiens, and T. Perez-Roux, Eds. Editions JFD, 2017.

[2] M. Pape, The art of driving: according to the guidelines of Benno von Achenbach. London: J.A. Allen, 1982.

[3] M. Secheppet, "Learning in a real and simulated training environment : articulations of experience in the activity of carriage driving learners," Ph.D. dissertation, University of Montpellier, 2020.

[4] J. J. Jouffroy, "L'analyse et la restitution des sensations par simulation en équitation : programme Persival," Science \& Sports, vol. 6, no. 2, pp. 129-131, 1991.

[5] P. Klavins, "The Equestrian Simulator Clergerie," http://simulateur.clergerie.free.fr/anglais.htm, 1993.

[6] M. Yamaguchi and N. Iguchi, "Development of a horseback riding simulator," Advanced Robotics, vol. 6, no. 4, pp. 517-528, 1991.

[7] Y. Amirat, C. Francois, G. Fried, J. Pontnau, and M. Dafaoui, "Design and control of a new six DOF parallel robot: Application to equestrian gait simulation," Mechatronics, vol. 6, no. 2, pp. 227-239, 1996.

[8] C.-W. Lee, S. G. Kim, and S. S. Na, "The effects of hippotherapy and a horse riding simulator on the balance of children with cerebral palsy," Journal of Physical Therapy Science, vol. 26, no. 3, pp. 423-425, 2014

[9] J.-H. Yoo, S.-E. Kim, M.-G. Lee, J.-J. Jin, J. Hong, Y.-T. Choi, M.-H. Kim, and Y.-S. Jee, "The effect of horse simulator riding on visual analogue scale, body composition and trunk strength in the patients with chronic low back pain," International Journal of Clinical Practice, vol. 68, no. 8, pp. 941-949, 2014.

[10] B. Vennetier, "Driving simulator for horse drawn vehicles," patent EP1 454 661A1, 2004.

[11] E. Encke, "Apparatus for learning to drive horse-drawn carriages," patent WO2 009026 999A1, 2009.

[12] H. M. Herr and T. A. McMahon, "A trotting horse model," The Int. Journal of Robotics Research, vol. 19, no. 6, pp. 566-581, 2000.

[13] Z. Bhatti, A. Shah, and F. Shahidi, "Procedural model of horse simulation," in Proceedings of the 12th ACM SIGGRAPH International Conference on Virtual-Reality Continuum and Its Applications in Industry, ser. VRCAI'13. ACM, 2013, pp. 139-146.

[14] P. M. Fitts, "The information capacity of the human motor system in controlling the amplitude of movement," Journal of Experimental Psychology, vol. 47, no. 6, pp. 381-391, 1954.

[15] S. Kim, Y. Koike, and M. Sato, "Tension Based 7 DOFs Force Feedback Device: SPIDAR-G," Transaction on Control Automation, and Systems Engineering, vol. 4, no. 1, pp. 9-16, 2002.

[16] L. Rebenitsch and C. Owen, "Review on cybersickness in applications and visual displays," Virtual Reality, vol. 20, pp. 101-125, 2016.

[17] J. Theureau, "Course-of-action analysis and course-of-action centered design," in Handbook of Cognitive Task Design, E. Hollnagel ed. Hillsdale (NJ): Lawrence Erlbaum, 2003, pp. 55-81.

[18] N. Mullen, J. Charlton, A. Devlin, and M. Bedard, "Simulator validity: Behaviors observed on the simulator and on the road," in Handbook of driving simulation for engineering, medicine, and psychology. CRC Press, 2011, pp. 1-18.

[19] S. Leblanc, "Analysis of video-based training approaches and professional development," Contemporary Issues in Technology and Teacher Education, vol. 18, no. 1, 2018.

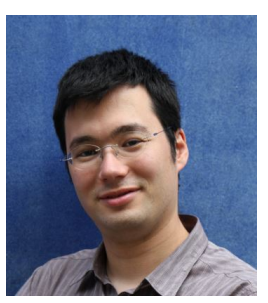

Jean-Rémy Chardonnet received his $\mathrm{Ph} . \mathrm{D}$. degree in robotics from the University of Montpellier II, France, in 2009. He was then with INRIA Grenoble, France, where he developed an awarded hands-on haptic interaction device for virtual manipulation. $\mathrm{He}$ is currently an Associate Professor at Arts et Métiers and LISPEN/Institut Image, Chalon-surSaône, France. His research interests include virtual reality and multimodal interaction, including human factors aspects. He is involved in different projects with industrial partners. He is a member of the IEEE.

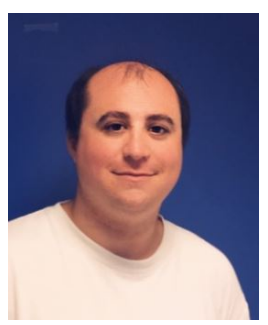

Julien Ryard received his Master degree in computer science from the University of Burgundy, France, in 2008. He is currently a project engineer at LISPEN/Institut Image, Chalon-sur-Saône, France. $\mathrm{He}$ is in charge of the development of software applications for immersive systems, as well as the maintenance of the virtual reality facilities of Institut Image. $\mathrm{He}$ is involved in different projects with industrial partners. He worked on the development of several training simulators for various fields of application, from healthcare to industry.

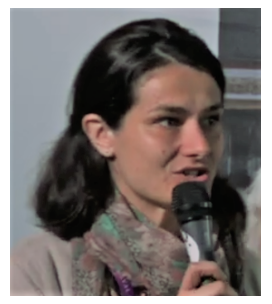

Mélanie Secheppet is a Ph.D. student at the University of Montpellier and LIRDEF, France. Her research work focuses on the analysis of trainees' experiences in real and simulated contexts using a situated cognitive anthropology approach. The issues relate to tracing the relationships emerging between these different experiences, with a focus on building them over a long period (several months). Trainees' transformations are studied from their own "world, body and culture".

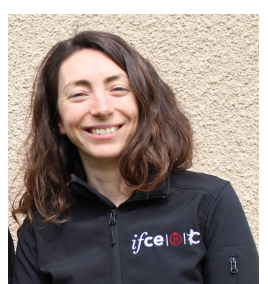

Clémence Bénézet is an engineer specializing in the fields of horse driving and equine traction at Ifce, France. She holds a Master degree in analysis and design in education and training. She has been working in partnership with the LIRDEF on research projects on educational innovation since 2016.

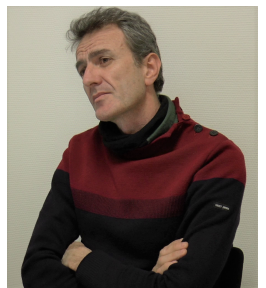

Guillaume Azéma is an Assistant Professor in education and training sciences at the University of Montpellier, France. He is specialized in the analysis of the activity of different categories of stakeholders (trainers, students, craftsmen, farmers, high-level athletes...), in interaction with other stakeholders, with physical or technical environments, and/or with animals. His research focuses on transforming work and/or training situations.

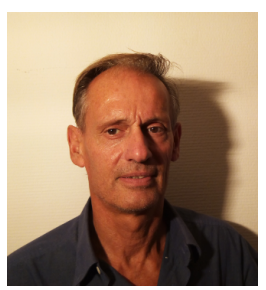

Serge Leblanc is a full professor in educational sciences at the University of Montpellier and LIRDEF, France. He develops programs of empirical and technological research for training. 